\title{
La investigación de servicios ecosistémicos en Chile: una revisión sistemática
}

\author{
Francisco De la Barrera ${ }^{1,2}$ fdelabarrera@uc.cl; Pamela Bachmann-Vargas ${ }^{2,3}$; Antonio \\ Tironi2 ${ }^{2,3}$
}

\section{RESUMEN}

La relación de los ecosistemas con los ecosistemas-sociedad y el bienestar humano, mediante el uso del concepto de servicios ecosistémicos, ha sido abordada con mayor énfasis en los últimos años, tanto en el ámbito científico, como también en el contexto de la gestión ambiental pública y la toma de decisiones. Un gran impulso para este interés lo constituyó la publicación en el año 2005 del reporte internacional Millenium Ecosystem Assessment (Evaluación de los Ecosistemas del Mileno). No obstante, a nivel de políticas e instrumentos de gestión ambiental el vínculo entre la investigación y la aplicación de los conocimientos es un aspecto aún pendiente, especialmente en países en desarrollo. Este trabajo hace una revisión sistemática de la investigación de servicios ecosistémicos en Chile, con el fin de detectar qué y cómo se estudian los servicios ecosistémicos en el país, de manera de servir para respaldar y orientar las necesidades futuras de investigación que puedan ser aplicadas en el contexto de la conservación de la biodiversidad, la gestión ambiental y la planificación territorial. Nuestros resultados muestran un importante incremento en el desarrollo de la investigación científica a partir de 1994, concentrándose especialmente luego de 2005, reflejando la tendencia internacional de incremento de trabajos sobre servicios ecosistémicos post-publicación del reporte ya mencionado. Tras el análisis se observa que los estudios realizados en Chile han utilizado mayoritariamente herramientas de valoración económica por sobre otro tipo de herramientas y que son escasos los ejercicios de modelación y mapeo de servicios ecosistémicos. Asimismo, hay una necesidad evidente de realizar estudios en ecosistemas desérticos (especialmente en aquellos costeros y altoandinos), en ecosistemas de montaña y glaciares, en ecosistemas antropizados de la zona centro-sur, siendo éstos grandes demandantes de servicios ecosistémicos provistos por los ecosistemas naturales cercanos, y en ecosistemas marino-costeros e insulares.

Palabras clave: ecosistemas, sociedad, gestión ambiental, toma de decisiones.

\section{Ecosystem services research in Chile: a systematic review}

\begin{abstract}
The relationship between ecosystems and human well-being, based on the ecosystem services approach, has been addressed with greater emphasis in recent years, both in science as well as in the context of public environmental management and decision-making. A major impetus for this interest was constituted by the publication in 2005 of the international report of the Millenium Ecosystem Assessment. However, in terms of policies and instruments for environmental management the link between research and application of knowledge is still a pending topic, especially in developing countries. This work makes a systematic review about ecosystem services research in Chile, in order to detect what and how ecosystem services are studied in the country. The goal is to help to support and guide future research needs to be enforced in the context of biodiversity conservation, environmental management and territorial planning. Our results show an important increasing in scientific research on the subject since 1994, especially after 2005, mirroring the international trend of increased ecosystem services research after the publication of the aforementioned report. The analyses show that studies in Chile have mostly used economic valuation tools, instead of modelling exercises or mapping of ecosystem services. Also, there is a clear need for studies in desert ecosystems (especially in costal and Andean zones), in mountain ecosystems and glaciers, in anthropogenic ecosystems of central-southern Chile (hotspots of demand of ecosystem services provided by natural ecosystems nearby) and in coastal and island ecosystems.
\end{abstract}

Keywords: ecosystems, society, environmental management, decision-making.

Recibido el 17 de agosto de 2015, aceptado el 5 de diciembre de 2015.

1 Instituto de Geografía \& Centro de Desarrollo Urbano Sustentable (CEDEUS), Pontificia Universidad Católica de Chile, Vicuña Mackenna 4860, Macul, Santiago, Chile.

2 Cienciambiental Consultores S.A., Santiago, Chile.

3 Fundación Centro Transdisciplinario de Estudios FES-Sistémicos (CTF), Santiago, Chile. 


\section{INTRODUCCIÓN}

Tras la publicación del reporte internacional MILLENIUM ECOSYSTEM ASSESSMENT (MEA 2005, Evaluación de Ecosistemas del Mileno), el entendimiento de la relación entre los ecosistemas y el bienestar humano, mediante el uso del concepto de servicios ecosistémicos, ha sido abordado con mayor énfasis en el ámbito científico, así como también en el contexto de la gestión pública, convirtiéndose en un nuevo paradigma para la conservación de la biodiversidad, la gestión ambiental y la planificación territorial (DE GROOT et al. 2010, WAINGER et al. 2010). Este nuevo paradigma engloba las interacciones socio-ecológicas de dependencia hombrenaturaleza, que contribuyen al bienestar de las personas y a las economías locales y nacionales, siendo de gran utilidad para evidenciar la sustentabilidad del paisaje, definida como la capacidad de proveer, consistentemente y en el largo plazo, servicios ecosistémicos esenciales para mantener y mejorar el bienestar humano (WU 2013).

El impulso con que se ha seguido el paradigma de los servicios ecosistémicos para la sustentabilidad enfrenta la dificultad de que existen muchas definiciones y aproximaciones, tanto disciplinares como metodológicas, para el estudio de los servicios ecosistémicos que son diferentes y no necesariamente complementarias (DAILY 1997, DE GROOT et al. 2002, MEA 2005, BOYD \& BANZHAF 2007, COWLING et al. 2008, DAILY et al. 2009, SUKHDEV et al. 2014), lo que afecta la integración y aplicación efectiva del concepto en la toma de decisiones.

Para la elaboración del reporte MEA (2005) se desarrollaron una serie de investigaciones en países de Latinoamérica tales como Argentina, Brasil, Chile, Colombia, Costa Rica y Perú (CAPISTRANO et al. 2005). En el caso de Chile, fueron analizados los servicios ecosistémicos de provisión de agua dulce y turismo de San Pedro de Atacama bajo perspectivas principalmente económicas (RIDES 2005).

Desde entonces, i.e. el año 2005, ha habido un importante aumento en las investigaciones científicas tanto a nivel global como latinoamericano (BALVANERA et al. 2012, DELGADO \& MARÍN 2015). A nivel nacional, es posible advertir un número importante de estudios enfocados en la provisión de ecosistemas forestales y de valoraciones económicas (OYARZÚN et al. 2005, NAHUELHUAL et al. 2007, LARA et al. 2009, LITTLE \& LARA 2010, DELGADO et al. 2013). Estudios de este tipo disponen de un amplio marco conceptual y de múltiples herramientas económicas para calcular distintos tipos de valores, especialmente para su monetarización. A escala nacional destacan trabajos sobre valoración económica de las áreas protegidas de Chile (FIGUEROA 2010, VÁZQUEZ et al. 2010).

En tanto, la incorporación de la dimensión geográfica en la evaluación de servicios ecosistémicos puede representarse a través de los siguientes atributos, los que serán abordados en este trabajo: (i) la espacialización de los servicios y de los ecosistemas proveedores de servicios (DELGADO \& MARÍN 2015), (ii) la elección de la escala espacial de análisis (MARTÍNEZ-HARMS \& BALVANERA 2012) y (iii) las implicancias de la evaluación de servicios ecosistémicos para la planificación territorial (NIEMELÄ et al. 2010, LATERRA et al. 2011).

Para una planificación y gestión integradas del territorio que conserven la biodiversidad a la vez que asegura el bienestar de la población, mediante una adecuada gestión ambiental y planificación territorial, es necesario disponer de conocimientos acerca de la provisión y distribución espacial de los servicios ecosistémicos, provistos tanto por ecosistemas terrestres como marinos-costeros. Al respecto, existen 
una serie de iniciativas internacionales tendientes a generar mapas y evaluaciones de distribución de servicios ecosistémicos, así como también para su incorporación en la toma de decisiones, especialmente en Europa con proyectos como Esmeralda (www.esmeralda-project.eu) y fondos de investigación como Horizon 2020. Se han desarrollado múltiples herramientas, en su mayoría computacionales, para integrar los servicios ecosistémicos a la toma de decisiones como: Natural Capital (www. naturalcapitalproject.org), ValuES (www. aboutvalues.net), ProEcoServ (www. proecoserv.cl) y GISCAME (www.giscame. com), entre otros.

Una evaluación de servicios ecosistémicos urbanos a nivel global encontró que sólo un $12,5 \%$ de las investigaciones publicadas utilizó algún modelo espacialmente explícito (GIS-based) para evaluar provisión y sólo un 3,3\% para evaluar demanda (HAASE et al. 2014). En Latinoamérica existen investigaciones que efectivamente han realizado mapeo de servicios ecosistémicos a diferentes escalas, incluso a nivel nacional y de largo plazo (ver BALVANERA et al. 2012). No obstante, pocos casos en Chile han incorporado el análisis de la distribución espacial de dichos servicios y son aún menos aquellos que han evaluado sus vínculos con la planificación territorial (BACHMANN-VARGAS 2013).

Desde una perspectiva normativa, el concepto de servicios ecosistémicos ha sido incorporado en la legislación chilena vigente a través de instrumentos regulatorios. Uno de ellos es el Reglamento de Evaluación de Impacto Ambiental (vigente desde diciembre de 2013), donde el artículo octavo, referente a localización y valor ambiental del territorio, indica en su párrafo sexto que: «se entenderá que un territorio cuenta con valor ambiental cuando corresponda a un territorio con nula o baja intervención antrópica y provea de servicios ecosistémicos locales relevantes para la población (...)». Otro instrumento es la Ley 20.283 de Recuperación y del Bosque Nativo y Fomento Forestal de 2008, la cual presenta una definición del concepto de servicios ambientales definiéndolos como «aquellos servicios que brindan los bosques nativos y las plantaciones que inciden directamente en la protección y mejoramiento del medio ambiente». Destaca, además, el Proyecto de Ley que crea el Servicio de Biodiversidad y Áreas Protegidas y el Sistema Nacional de Áreas Protegidas, el cual define los servicios ecosistémicos como «la contribución directa o indirecta de los ecosistemas al bienestar humano». Finalmente, es posible encontrar el concepto de servicios ecosistémicos en estrategias de desarrollo regional (ILPESCEPAL/DIPLADE AYSÉN 2009), así como también dentro del manual de certificación ambiental municipal (JARA et al. 2014). Actualmente, el Ministerio del Medio Ambiente cuenta con tres ejes programáticos con el objetivo de incorporar el valor de la biodiversidad y los servicios ecosistémicos en la toma de decisiones públicas y privadas.

En este contexto, RUCKELSHAUS et al. (2015) establecen que existen cuatro etapas para integrar biodiversidad y ecosistemas de manera efectiva: (i) realizar investigación, (ii) cambiar perspectivas, (iii) generar acción y (iv) producir resultados. Estas cuatro vías son paralelas, complementarias y su capacidad de impacto en la toma de decisiones es ascendente. La primera etapa es fundamental, sobre todo en países en vías de desarrollo donde es escasa la información científica y técnica que sirva para la realización de evaluaciones a nivel nacional o regional y para la toma de decisiones.

Este trabajo desarrolla una revisión sistemática de la investigación realizada sobre servicios ecosistémicos en Chile, a través de análisis bibliométricos (HAASE et al. 2014, TANCOIGNE et al. 2014), incorporando una orientación geográfica. Esta investigación representa el primer 
esfuerzo sistemático por recopilar y evaluar la bibliografía publicada en Chile, con el fin de servir de respaldo y orientación para las futuras investigaciones que contribuyan a la conservación de la biodiversidad, la gestión ambiental y la planificación territorial mediante la investigación de servicios ecosistémicos, a nivel local, regional y/o nacional.

\section{MATERIALES Y MÉTODOS}

\section{Revisión bibliográfica}

Se realizó una búsqueda exhaustiva de documentos disponibles en medios digitales durante el mes de noviembre de 2014. Los documentos fueron localizados a través del buscador académico Google Scholar, en base a las siguientes palabras clave, tanto en español como en inglés, y de manera combinada: servicios ecosistémicos de Chile, servicios ambientales, mapeo de servicios ecosistémicos, valoración económica, capital natural, valoración económica de áreas protegidas, entre otras. Se utilizó Google Scholar con el objetivo de abarcar diversos tipos de publicaciones, no sólo los artículos científicos.

\section{Sistematización de documentos}

Los documentos recopilados fueron agrupados en tres categorías: artículos científicos, tesis y otros (e.g. informes o reportes). Posteriormente, todos los documentos fueron sistematizados en una base de datos, clasificando cada documento en términos de: (i) autoría, (ii) año de publicación, (iii) revista donde se publicó e (iv) indexación (Science Citation Index, ISI) según el registro de THOMSON REUTERS (2013). Asimismo, para su caracterización geográfica se registró: (i) qué regiones administrativas de Chile son parte del área de estudio, donde se asignó la categoría de varias a las investigaciones que involucraron más de cuatro regiones, (ii) la escala de estudio, siendo local si el estudio fue espacialmente muy acotado; regional, si fue más amplio; nacional y no aplica, para estudios de opinión, y (iii) coordenadas geográficas. Otros datos generales de cada investigación fueron: (i) idioma original de la publicación, (ii) fuente de financiamiento, i.e. público, privado, mixto (público y privado) y otro, si corresponde a fondos internacionales.

Respecto al tipo de investigación, cada documento se clasificó según su tipología y objetivo de estudio: artículo científico empírico; consultoría; diseminación; opinión; revisión; tesis de pregrado; tesis de postgrado y otro. Se entendió como artículo científico empírico a las investigaciones que se basaron en información primaria o secundaria para generar nuevos resultados a través de la aplicación de diversos métodos de investigación, ya sean cualitativos o cuantitativos. Por consultoría se entendió a los informes de consultorías, ya sea para organismos públicos o privados. Por diseminación se definieron a los trabajos publicados que presentaron un análisis descriptivo orientado a todo público, en un formato magazine. Los documentos identificados de opinión correspondieron a aquellos que presentaron una crítica respecto a un tema, también orientado a todo público y que a su vez señalaron en el documento que corresponden a una opinión. Los documentos clasificados en la categoría de revisión incluyen análisis del estado del arte, revisión de conceptos, etc. Los documentos de las categorías de pre y post grado se clasifican en base a su identificación en el mismo documento. Se añadió además la categoría otros, en caso de no poder clasificar algún trabajo en las categorías antes señaladas.

Se identificó el tipo de ecosistema donde se focalizó cada investigación: terrestre, acuático continental, marino-costero o bien no aplica. Por otro lado, se detalló si los servicios ecosistémicos analizados fueron mapeados, modelados $\mathrm{y} / \mathrm{o}$ valorizados. Al respecto, se entendió como mapeo de 
servicios ecosistémicos a la generación de mapas de distribución de éstos, que pueden haber sido generados sobre la base de equivalencias entre, por ejemplo, mapas de distribución de bosque, donde se pueden construir mapas proxy del servicio de regulación global del clima; se incluyó también dentro de esta categoría a los mapas de zonas de provisión y de beneficiarios, y a la generación de mapas propios con la aplicación de diversos indicadores. En tanto, para registrar las investigaciones que realizaron una modelación de servicios ecosistémicos, se consideraron aquellas que a partir de un modelo numérico (espacializado o no) calcularon los valores de los indicadores (o flujos biofísicos) de los servicios ecosistémicos estudiados. Es importante aclarar que la modelación de servicios ecosistémicos no necesariamente genera un mapa, así, por ejemplo, la modelación de productividad hídrica es realizada en base a modelos hidrológicos y no arroja un mapa. Finalmente, para evaluar si la investigación realizó valoración económica, se consideró la monetarización de los servicios ecosistémicos, ya sea en base a fuentes primarias o secundarias de información. Se registró la moneda original y el año de valoración, así como también el valor económico estimado o calculado de manera directa en dólares americanos (USD). Se identificó además el tipo de valor utilizado para la valorización de servicios ecosistémicos (valor de uso, valor de no uso, valor de uso-valor de no uso y no aplica). Los métodos de valoración económica señalados en las investigaciones fueron sistematizados según la clasificación elaborada por TEEB (2010: 44).

\section{Análisis bibliométricos}

Los análisis bibliométricos son un método de investigación bibliográfica que pueden abarcar desde el análisis de citas bibliográficas (quién cita y quién es citado por quién), hasta la valoración de la productividad de la investigación y el análisis de la evolución de los diversos campos científicos a través de indicadores cuantitativos (BLOUIN et al. 2013, YOUNG \& WOLF 2006). De este modo, la base de datos de investigaciones realizadas en Chile fue analizada cuantitativamente a partir de sus atributos, describiendo: (i) cantidad y tipo de documentos, (ii) aspectos asociados a la autoría, afiliación y fuentes de financiamiento, (iii) productividad anual, (iv) medios (revistas) en los cuales han sido publicadas las investigaciones, (v) aspectos geográficos específicos (localización por región y escalas de estudio) y (vi) los servicios ecosistémicos más estudiados, junto con las técnicas utilizadas (mapeo, modelación, valorización).

Es importante señalar que los servicios ecosistémicos fueron identificados y homologados a la clasificación internacional de servicios ecosistémicos, llamada CICES por sus siglas en inglés (The Common International Classification of Ecosystem Services; HAYNES-YOUNG \& POTSCHIN 2013), la cual es utilizada por el Ministerio del Medio Ambiente de Chile. Dicha clasificación presenta un listado de 48 servicios ecosistémicos organizados por división, sección y grupo, los cuales se encuentran divididos en 3 categorías: provisión, regulación y culturales. Los servicios ecosistémicos de provisión corresponden a todos los productos tangibles de los ecosistemas y que son utilizados por los seres humanos, ya sea para nutrición, obtención de materias primas, generación de energía, etc. Estos productos suelen ser comercializados y consumidos directamente (e.g. leña, madera, frutos silvestres, etc.). Por su parte, los servicios ecosistémicos de regulación se refieren a los beneficios que se obtienen de los ecosistemas, o bien a la contribución de los ecosistemas al bienestar de las personas, a través de la regulación de procesos naturales, tales como purificación del agua, control de la erosión, captura de carbono, entre otros (KANDZIORA et al. 2013). Finalmente, los servicios ecosistémicos culturales corresponden a los beneficios no 
materiales que las personas obtienen desde los ecosistemas a través de actividades espirituales, desarrollo cognitivo, reflexión y recreación, incluyendo el desarrollo de conocimiento, relaciones sociales y valores estéticos (MEA 2005). De este modo, la naturaleza resulta una fuente de inspiración científica, cultural y artística, la cual a su vez provee innumerables oportunidades para la educación y la investigación científica y social (DE GROOT et al. 2002).

\section{RESULTADOS}

La revisión bibliográfica arrojó un total de 105 documentos, de los cuales 61 son artículos científicos, 33 son tesis y 11 fueron considerados en la categoría de otros. La sistematización de los documentos alcanzó un total de 4.138 registros en la base de datos, la cual puede ser consultada en el sitio web del Ministerio del Medio Ambiente (http://portal.mma. gob.cl/servicios-ecosistemicos/), así como también en el análisis detallado de cada categoría presentado en el informe técnico.

\section{Cantidad y tipo de documentos}

Del total de artículos científicos, 49 presentan desarrollo empírico y 16 realizan revisiones. Un número menor corresponde a documentos de consultoría (4), diseminación (1) y opinión (2). Un 31\% (33 artículos) fue publicado en revistas ISI. Otro porcentaje similar está representado por documentos de tesis, 15 de pregrado y 18 de postgrado (Fig. 1).

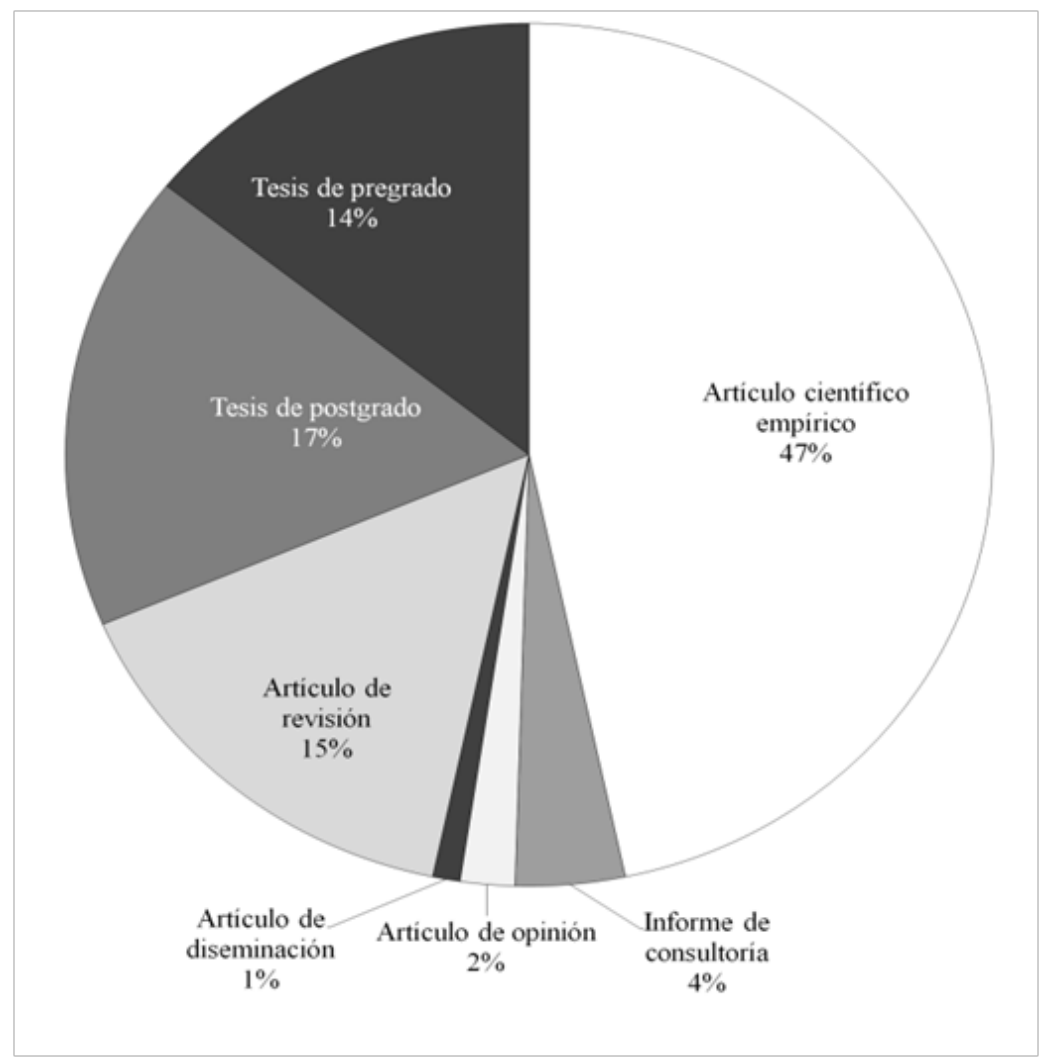

Fig. 1. Tipo de documentos con investigaciones SObre Servicios ecosistémicos en Chile.

Fig. 1. Type of documents With Research about eCosystem SERVICES CONdUCted in Chile. 


\section{Autoría y fuentes de financiamiento}

Más de 150 autores (personas naturales e instituciones) han publicado artículos sobre servicios ecosistémicos en Chile. De éstos, 84 han publicado un total de 33 artículos ISI. En promedio, los documentos tienen 3 autores por artículo. No obstante, si se consideran sólo los artículos ISI, este promedio se eleva a 4 autores por artículo. Sólo 6 autores han publicado más de 5 artículos y solamente 8 investigadores tienen más de 3 artículos ISI. Estos investigadores se concentran en las ciudades de Santiago, Valdivia, Concepción y Antofagasta.
En relación a la fuente de financiamiento, más de un $40 \%$ no la informa. Entre aquellos artículos que sí informan su fuente de financiamiento, un $30 \%$ declara financiamiento público y un $13 \%$ un financiamiento mixto (público y privado a la vez). Sólo cuatro artículos (3\% del total de documentos) tuvieron financiamiento privado. Once artículos indicaron otra fuente de financiamiento, compuesta principalmente por fondos internacionales, ya sea a través de proyectos o becas de pre y postgrado.

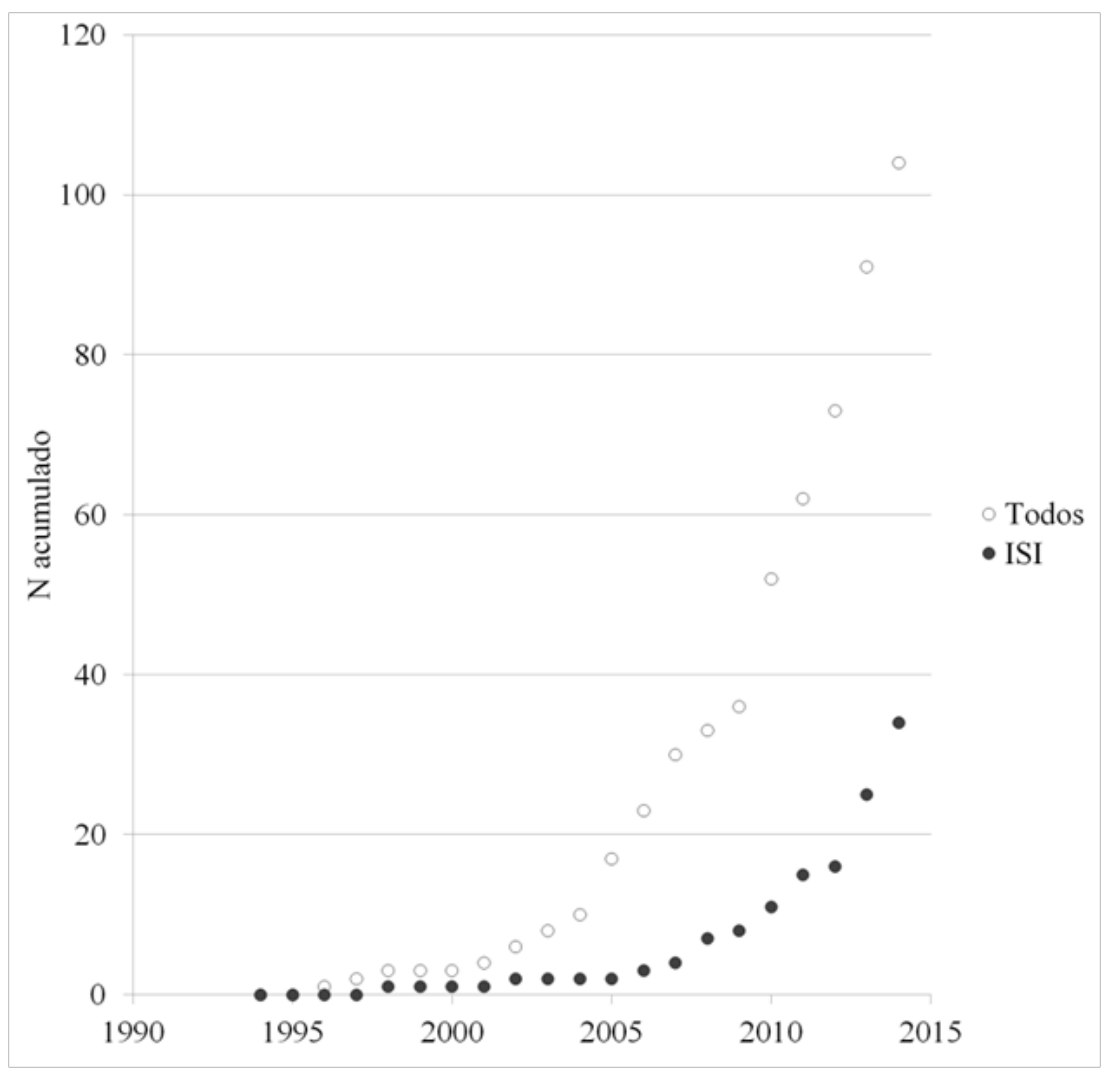

Fig. 2. Productividad científica acumulada sobre Servicios ecosistémicos en Chile.

Fig. 2. Accumulated scientific Productivity about ecosystem Services in Chile. 


\section{Productividad anual}

El número de documentos se ha incrementado paulatinamente a partir de 1994. En la Figura 2 se pueden observar tres períodos dentro de los últimos 20 años. Un primer período (1994-2004), previo a la publicación de MEA (2005), presenta una tasa de producción de un artículo por año (diez en total), siendo sólo dos de ellos ISI. Luego, se identifica un segundo período posterior a la publicación de MEA (2005; 2005-2009), en el que se publicaron 26 documentos, es decir 5,2 artículos al año, quintuplicando la cantidad de artículos anuales publicados en el periodo anterior y elevando de dos a ocho la productividad de artículos ISI. El siguiente período está representado entre 2010 y 2014, donde la productividad fue de 13,6 artículos por año (68 documentos), casi triplicando la producción de la etapa inmediatamente anterior, mientras que la productividad en revistas ISI también se triplicó, aumentando de 8 a 34 .

\section{Medios de difusión}

Son 41 las revistas que han sido utilizadas para publicar trabajos científicos, de las cuales 23 se encuentran indexadas en ISI (THOMSON REUTERS 2013). Todas las revistas utilizadas son de editoriales internacionales, a excepción de la revista Bosque, de producción nacional (ISI, I.F.= $0,3955)$. Esta es la revista que contiene más artículos sobre investigaciones de servicios ecosistémicos en Chile, con cuatro artículos, le sigue Environment and Development Economics (ISI, I.F. $=0,9856$ ) con tres artículos, mismo número que la revista nacional Ambiente y Desarrollo. Luego, ocho revistas contienen dos artículos, seis de las cuales son ISI: AMBIO, Ecological
Economics, Environmental Monitoring and Assessment, Journal for Nature Conservation, Journal of Environmental Management y Landscape Ecology. Las otras dos revistas son Ecosystem Services y Gestión Ambiental.

\section{Localización y escala geográfica}

La región con más investigaciones es Valparaíso, seguido por Los Ríos, Metropolitana de Santiago, Los Lagos, Magallanes y Aysén (Fig. 3). Una gran parte de las investigaciones cubren más de una región, no obstante predominan las investigaciones a escala local (67 artículos; $64 \%$ ) por sobre investigaciones a nivel regional o nacional (11 y 13 artículos respectivamente).

Respecto de la distribución espacial, es posible advertir una gran concentración en la zona central y centro sur, especialmente en Valdivia, que existe investigación en todas las regiones y que hay vacíos de información en: (i) ecosistemas desérticos, desde la costa al altiplano, (ii) zonas montañosas y glaciares, a lo largo de todo Chile, (iii) ecosistemas de origen antrópico, tales como ecosistemas urbanos, plantaciones forestales y agroecosistemas (e.g. regiones del Maule y de la Araucanía) $\mathrm{y}$ (iv) ecosistemas marinos-costeros $\mathrm{e}$ insulares.

En general, casi todos los artículos (98 de 105) declaran el tipo de ecosistema evaluado, siendo los ecosistemas terrestres los que han recibido mayor atención (77). Por el contrario, los ecosistemas acuáticos continentales y marinos-costeros han sido muy escasamente estudiados, con 10 y 11 registros respectivamente. 


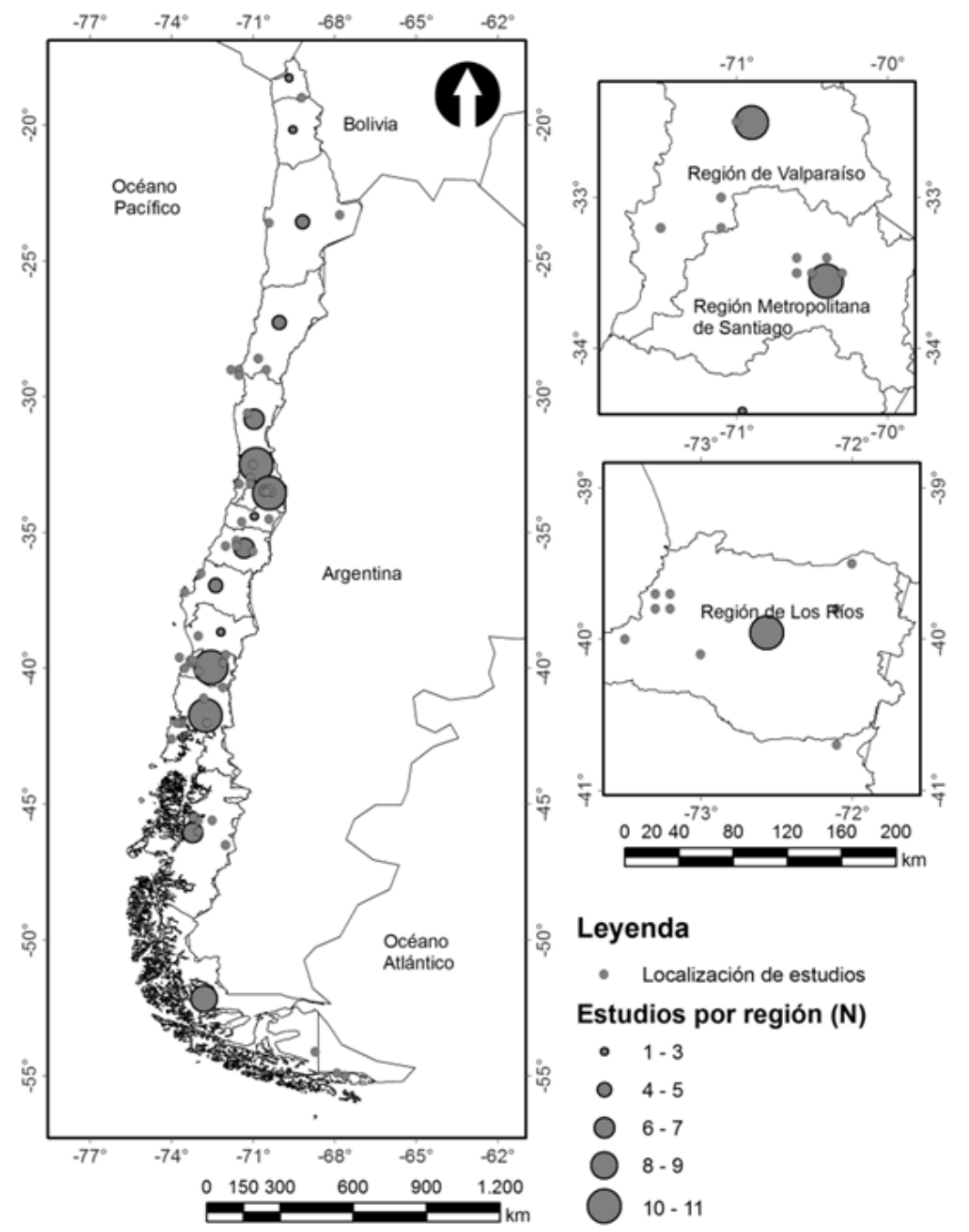

Fig. 3. Distribución espacial de estudios sobre Servicios ecosistémicos en Chile. La LOCALIZACIÓN DE LAS INVESTIGACIONES ES APROXIMADA Y REPRESENTA EL CENTROIDE DEL ÁREA DE ESTUDIO, LA QUE SE PUEDE EXTENDER A MÁS DE UNA REGIÓN ADMINISTRATIVA.

Fig. 3. Geographic location of WORKS ABOUt ECOSYSTEM SERVICES. The LOCATION IS APProXimate AND REPRESENTS THE CENTROID OF THE STUDY AREA, WHICH MAY BE EXTENDED MORE THAN ONE ADMINISTRATIVE REGION.

\section{Servicios ecosistémicos más estudiados y técnicas utilizadas}

Los 10 servicios ecosistémicos más estudiados, homologados a la clasificación CICES, se muestran en la Figura 4. Al respecto, es posible señalar que estos servicios ecosistémicos corresponden principalmente a aquellos relacionados con el turismo, ya sea a través de actividades recreativas que incorporan actividades físicas o a través de experiencias tales como observación de aves. También resulta importante el estudio de la provisión de agua dulce para beber y otros usos. 
De los 105 documentos analizados, 10 realizanunmapeodeserviciosecosistémicos, 11 realizan modelación de servicios ecosistémicos y 58 realizan valoración económica de servicios ecosistémicos, siendo el aspecto más publicado en esta temática. Estas investigaciones se realizaron preferentemente en ecosistemas terrestres y sólo una de cada 10 investigaciones en cada temática se realizó en ecosistemas acuáticos continentales o marino-costeros.

Condiciones químicas del agua dulce

Estético

Fibras y otros materiales de plantas, algas y animales para uso directo o ser procesados

Regulación global del clima por la reducción de las concentraciones de gases de efecto invernadero

Ciclo hidrológico y mantención del flujo de agua

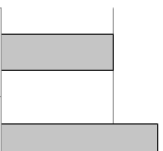

Existencia

Agua superficial para otros usos

Agua superficial para beber

Uso físico del paisaje en diferentes condiciones ambientales

Uso experiencial de plantas, animales y paisajes en diferentes condiciones ambientales
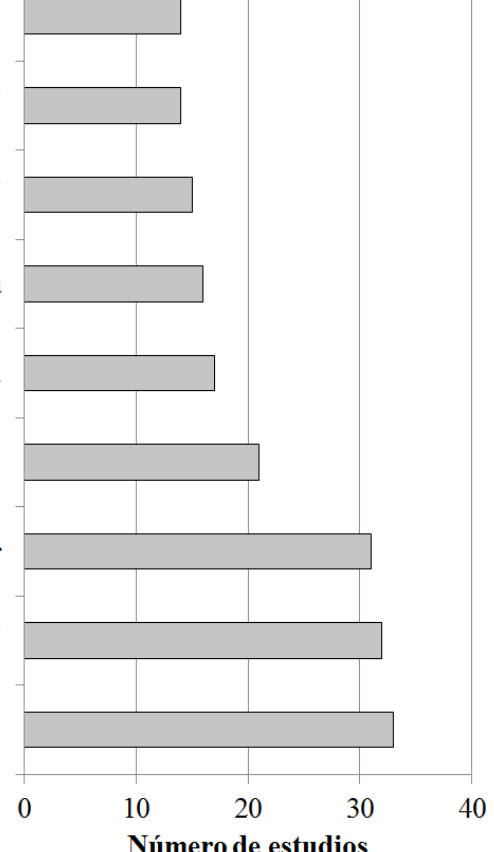

Número de estudios

Fig. 4. Servicios ecosistémicos más estudiados en Chile, según Clasificación CiCES.

Fig. 4. The most studied ecosystem Services in Chile, according to CiCES Classification.

\section{DISCUSIÓN}

Una revisión bibliográfica en temas de investigación en permanente crecimiento, como lo es la investigación de servicios ecosistémicos, es un proceso iterativo que debe repetirse y actualizarse con frecuencia, ya que la cantidad de documentos a incorporar aumenta año a año y trae consigo un cambio en los patrones de lo que se investiga. El esfuerzo y éxito en la recopilación de documentos en formato digital se relacionan directamente con el tipo de documento, siendo más fácil acceder a artículos científicos en formato digital. No ocurre lo mismo con documentos como tesis, informes de proyectos $\mathrm{y} / \mathrm{o}$ consultorías, por lo que el análisis aquí mostrado podría sub-representarlos, teniendo en cuenta que esta investigación sólo consideró medios digitales. 
La única revista nacional ISI que ha publicado artículos en la temática es la revista Bosque, la cual ingresó al ISI Web of Science en junio de 2009. Existen otras revistas nacionales ISI que por su temática general podrían tener artículos sobre servicios ecosistémicos (e.g. Revista de Geografía Norte Grande o Revista Chilena de Historia Natural), no obstante los autores han preferido enviar sus trabajos a otras revistas internacionales ISI y/o a revistas nacionales indexadas en SCOPUS o LATINDEX.

Por otro lado, el mapeo y/o modelación de servicios ecosistémicos se encuentra escasamente desarrollado en Chile, con 15 y 16 trabajos cada uno, lo cual no es la excepción dentro del contexto latinoamericano. De acuerdo a la revisión realizada por CROSSMAN et al. (2013) existen sólo 3 artículos ISI sobre mapeo en Latinoamérica, mientras en Europa hay 47. El número reportado en este trabajo es mayor porque se incluyó otro tipo de documentos. Esto expone la carencia de conocimientos acerca de cómo la provisión y la demanda de servicios ecosistémicos se encuentra espacialmente distribuida en diferentes escalas geográficas, en contraste a mayores conocimientos acerca de su valor económico.

Por su parte, los ecosistemas terrestres son los más estudiados, destacando las investigaciones a nivel de cuencas, en el bosque templado lluvioso, en el matorral esclerófilo y en humedales, entre otros, así como también investigaciones específicas en áreas naturales protegidas. Respecto a la distribución espacial en Chile, los resultados indican una mayor frecuencia de investigaciones en las regiones centrales y en localidades específicas de las regiones australes. De esto se desprende que las zonas menos estudiadas son la norte, en especial los ecosistemas desérticos, y la centro-sur, donde es escaso el conocimiento sobre paisajes y donde interactúan ecosistemas naturales y antropizados (e.g. bosques, plantaciones forestales, matorrales y praderas agrícolas). Adicionalmente, la investigación de servicios ecosistémicos en ambientes urbanos se encuentra escasamente representada.

Algunos servicios públicos han licitado investigaciones sobre servicios ecosistémicos que han contribuido a la investigación permanente en Chile, principalmente en formato de consultorías. Algunos de estos servicios son la Subsecretaría de Pesca (CENRE 2015), el Servicio Agrícola y Ganadero (MORALES et al. 2011) y el Ministerio de Medio Ambiente (e.g. FORECOS 2014). Sin embargo, las fuentes de financiamiento para este tipo de estudios aún son escasas y en general las investigaciones realizadas responden a financiamiento de tipo no regular.

Comoseha expuestoalolargo de estarevisión, el concepto de servicios ecosistémicos ha sido abordado principalmente mediante las valoraciones económicas y a su vez ha sido incorporado como un nuevo término en la agenda de la gestión ambiental pública. Sin embargo, no existe evidencia de que su monetarización haya sido incorporada de manera efectiva en la toma de decisiones ambientales a nivel nacional.

La aplicación de los resultados obtenidos en diferentes investigaciones efectuadas en Chile, para el contexto de la conservación de la biodiversidad, la gestión ambiental y la planificación territorial, es reducida, principalmente dada la acotada escala de los estudios -especialmente locales- y sus alcances limitados -pocos servicios evaluados-, que dificultan incluso su aplicación en instrumentos propios de los gobiernos locales (e.g. instrumentos de planificación territorial o de desarrollo comunal). Dentro de éstos destacan las investigaciones a escala nacional (14), que sin duda aportan al diagnóstico de todo el territorio.

Un tema pendiente es contar con una clasificación consensuada de ecosistemas 
terrestres y marinos-costeros a nivel nacional, dado que cada investigación los aborda de manera diferente y usando clasificaciones y delimitaciones que pueden resultar confusas, ya que existen servicios cuyo origen y escala espacial pueden sobrepasar esas categorías. Al respecto, si bien el Ministerio del Medio Ambiente define que los pisos vegetacionales son representativos de los ecosistemas terrestres de Chile (PLISCOFF \& LUBERT 2006), éstos no necesariamente son útiles para estudiar servicios ecosistémicos, por ser un modelo potencial de la fisionomía y la composición de especies, excluyendo ecosistemas antropizados. Al respecto, los autores de investigaciones sobre servicios ecosistémicos en Chile han preferido utilizar el Catastro del Bosque Nativo y Usos de Suelo, elaborado por la Corporación Nacional Forestal (CONAF), o bien utilizar clasificaciones ad hoc, creadas para el propósito de la evaluación. Algo similar ocurre con los ecosistemas marinos, donde la clasificación depende del punto de vista (FARIÑA et al. 2006). En Europa se acordó hace más de 30 años una clasificación de los ecosistemas denominada CORINE Land Cover, que integra 44 clases, basada tanto en la cobertura como en el uso del suelo. Esta cobertura es actualizada periódicamente y ha sido el insumo básico para diferentes evaluaciones de servicios ecosistémicos (MAES et al. 2011, BURKHARD et al. 2012, KOSCHKE et al. 2012).

La predominancia de investigaciones de valoración económica se relaciona directamente con el marco conceptual que afirma que la monetarización de los servicios ecosistémicos es un elemento esencial que debe ser incorporado en la toma de decisiones (ATKINSON et al. 2012), bajo el paradigma del análisis costobeneficio. No obstante, los resultados de esta revisión muestran que la variabilidad encontrada en los métodos de valoración económica utilizados y la discrecionalidad en la delimitación de los ecosistemas hacen compleja la agregación de los resultados de distintas investigaciones de valoración económica. En este sentido, es necesario el desarrollo de un nuevo marco conceptual donde la contabilidad de los beneficios que la sociedad percibe desde los ecosistemas incorpore no sólo aspectos monetarios, si no que utilice una concepción más integral de bienestar y calidad de vida, incorporando además aspectos sociales y culturales (HAINES-YOUNG \& POTSCHIN 2009, CHAN et al. 2012).

\section{CONCLUSIONES}

En Chile se sigue la tendencia de investigar cada vez más los servicios ecosistémicos provistos por sus ecosistemas tanto a través de proyectos de investigación que resultan en publicaciones científicas, como también a través de tesis de pre y postgrado y mediante consultorías. Esta tendencia al alza ha sido la respuesta al hito de la publicación del reporte MEA (2005), como ha ocurrido también en otros países (DELGADO \& MARÍN 2015). Las investigaciones son desarrolladas principalmente en cinco regiones administrativas cercanas a dos ciudades (Santiago y Valdivia) donde se localizan grandes universidades que concentran investigadores en servicios ecosistémicos. Al respecto, es llamativa la escasa investigación en las regiones del Maule, Biobío y la Araucanía, que cuentan con importantes universidades y centros de investigación. Los ecosistemas de montaña (andina y costera) han sido poco investigados, siendo valiosos por su importancia en la provisión de servicios ecosistémicos hacia ecosistemas antropizados, los que también han sido poco estudiados y en efecto pueden proveer de servicios ecosistémicos, ayudando a disminuir la demanda sobre los ecosistemas naturales. Finalmente, la mayor utilización de herramientas de valoración económica asociadas a una perspectiva de costobeneficio ha sido ampliamente preferida por los tomadores de decisiones. Por ende, es necesario avanzar hacia una perspectiva 
nueva o paradigma que contribuya a una mejor planificación y gestión integrada del territorio, que permita la conservación de la biodiversidad y asegure, de manera consistente y en el largo plazo, la provisión de servicios ecosistémicos esenciales para mantener y mejorar el bienestar humano. Para lograr este cambio se requiere contar con conocimientos acerca de qué servicios ecosistémicos son provistos por un ecosistema dado y cuál es su valor económico (aproximado), pero también es importante conocer cómo varían en el tiempo y en el espacio y cuáles son las relaciones estructurales y funcionales entre ecosistemas vecinos, siendo fundamentales las investigaciones que incorporen modelación y mapeo de servicios ecosistémicos.

\section{AGRADECIMIENTOS}

Este trabajo fue financiado parcialmente a través de fondos públicos del Ministerio de Medio Ambiente (Chile). Los autores agradecen a FONDECYT Postdoctorado $3150403 / 2015$ y $3130463 / 2013$ y a BecasChile Doctorado 72150473/2014.

\section{REFERENCIAS}

ATKINSON, G., I. BATEMAN \& S. MOURATO, 2012. Recent advances in the valuation of ecosystem services and biodiversity. Oxford Review of Economic Policy, 28(1): 22-47.

BACHMANN-VARGAS, P., 2013. Ecosystem services modeling as a tool for ecosystem assessment and support for decision making process in Aysén region, Chile (Northern Patagonia). Master thesis. Faculty of Agriculture and Nutritional Sciences, Christian-Albrechts-Universität, Kiel, Germany.
BALVANERA, P., M. URIARTE, L. ALMEIDA-LEÑERO, A. ALTESOR, F. DE CLERCK, T. GARDNER, J. HALL, A. LARA, P. LATERRA, M. PEÑACAROS, D.M. SILVA MATOS, A.L. VOGL, L.P. ROMERO-DUQUE, L.F. ARREOLA, A.P. CARO-BORRERO, F. GALLEGO, M. JAIN, C. LITTLE, R.O. XAVIER, J.M. PARUELO, J.E. PEINADO, L. POORTER, N. ASCARRUNZ, F. CORREA, M.B. CUNHA-SANTINO, A.P. HENÁNDEZSÁNCHEZ \& M. VALLEJOS, 2012. Ecosystem services research in Latin America: The state of the art. Ecosystem Services, 2: 56-70.

BLOUIN, M., N. SERY, D. CLUZEAU, J.J. BRUN \& A. BÉDÉCARRATS, 2013. Balkanized research in ecological engineering revealed by a bibliometric analysis of earthworms and ecosystem services. Environmental Management, 52: 309-320.

BOYD, J. \& S. BANZHAF, 2007. What are ecosystem services? The need for standardized environmental accounting units. Ecological Economics, 63: 616-626.

BURKHARD, B., F. KROLL, S. NEDKOV \& F. MÜLLER, 2012. Mapping ecosystem service supply, demand and budgets. Ecological Indicators, 21, 17-29.

CAPISTRANO, D., C. SAMPER, M.J. LEE \& C. RAUDSEPP-HEARNE (Eds.), 2005. Ecosystems and human well-being: multiscale assessments, Vol. 4. Findings of the Sub-global Assessments Working Group of the Millennium Ecosystem Assessment. Disponible en: http://www.unep.org/ maweb/documents/document.773.aspx.pdf

CENRE 2015. Valoración económica de los servicios ecosistémicos asociados a los recursos hídricos bajo la Ley General de Pesca y Acuicultura de la región de Aysén. Informe de Avance, junio de 2015. Proyecto FIP N ${ }^{\circ} 2014-85$, preparado para la Subsecretaría de Pesca y Acuicultura. 
CHAN, K.M.A., T. SATTERFIELD \& J. GOLDSTEIN, 2012. Rethinking ecosystem services to better address and navigate cultural values. Ecological Economics, 74: 8-18.

COWLING, R.M., B. EGOH, A.T. KNIGHT, P.J. O'FARREL, B. REYERS, M. ROUGET, D.J. ROUX, A. WELS \& A. WILHEM-RECHMAN, 2008. An operational model for mainstreaming ecosystem services for implementation. PNAS 105(28): 9483-9488.

CROSSMAN, N.V., B. BURKHARD, S. NEDKOV, L. WILLEMEN, $K$. PETZ, I. PALOMO, E.G. DRAKOU, B. MARTÍN-LÓPEZ, T. MCPHEARSON, K. BOYANOVA, R. ALKEMADE, B. EGOH, M.B. DUNBAR \& J. MAES, 2013. A blue print for mapping and modeling ecosystem services. Ecosystem Services, 4: 4-14.

DAILY, G., 1997. Nature's services: societal dependence on natural ecosystems. Island Press.

DAILY, G., S. POLASKY, J. GOLDSTEIN, P.M. KAREIVA, H.A. MOONEY, L. PEJCHAR, T.H. RICKETTS, J. SALZMAN \& R. SHALLENBERGER, 2009. Ecosystem services in decision making: time to deliver. Frontiers in Ecology and the Environment, 7(1): 21-28.

DE GROOT, R.S., M.A. WILSON \& R.M.J. BOUMANS, 2002. A typology for the classification, description and valuation of ecosystem functions, goods and services. Ecological Economics, 41: 393-408.

DE GROOT, R.S., R. ALKEMADE, L. BRAAT, L. HEIN \& L. WILLEMEN, 2010. Challenges in integrating the concept of ecosystem services and values in landscape planning, management and decision making. Ecological Complexity, 7: 260-272.
DELGADO, L.E., M.B. SEPÚLVEDA \& V.H. MARÍN, 2013. Provision of ecosystem services by the Aysén watershed, Chilean Patagonia, to rural households. Ecosystem Services, 5: 102-109.

DELGADO, L.E. \& V.H. MARÍN, 2015. Ecosystem services: Where on earth? Ecosystem Services, 14: 24-26.

FARIÑA, J.M., P.G. OSSA \& J.C. CASTILLA, 2006. Diversidad de ecosistemas: Ecosistemas marinos. En: SABALL, A. Biodiversidad de Chile: patrimonio y desafíos. Comisión Nacional del Medio Ambiente, 96-105.

FIGUEROA, E., 2010. Valoración económica detallada de las áreas protegidas de Chile. Proyecto GEF-MMA-PNUD Creación de un Sistema Nacional Integral de Áreas Protegidas para Chile. Disponible en: http://www.proyectogefareasprotegidas.cl/ wp-content/uploads/2011/09/valoracion $\% 20$ economica.ok.24-05-2011.pdf

FORECOS, 2014. Informe final de consultoría Recopilación de Información de Indicadores de Servicios Ecosistémicos a Nivel Nacional e Internacional. Ministerio de Medio Ambiente, Chile.

HAASE, D., N. LARONDELLE, E. ANDERSSON, M. ARTMANN, S. BORGSTRÖM, J. BREUSTE, E. GÓMEZ-BAGGETHUN, A. GREN, Z. HAMSTEAD,R.HANSEN,N.KABISCH, P. KREMER, J. LAGEMEYER, E. RALL, T. MCPHEARSON, S. PAULEIT, S. QURESHI, N. SCHWARZ, A. VOIGT, D. WURSTER \& T. ELMQVIST, 2014. A quantitative review of urban ecosystem service assessments: concepts, models, and implementation. Ambio, 43: 413-433.

HAYNES-YOUNG, R. \& M. POTSCHIN, 2013. Common International Classification of Ecosystem Services (CICES): Consultation on Version 4, August-December 2012. Report to the 
European Environment Agency. Disponible en: http://test.matth.eu/content/uploads/ sites/8/2012/07/CICES-V43_RevisedFinal_Report_29012013.pdf

ILPES-CEPAL/DIPLADE Aysén, 2009. Estrategia de Desarrollo Regional, período 2009-2030. División de Planificación y Desarrollo del Gobierno de Aysén (DIPLADE). Disponible en: http:// www.subdere.gov.cl/documentacion/ aysen-estrategia-de-desarrollo-regionalperiodo-2009-2030

JARA, C., M.A. SANTOS, M.J. ESQUIVEL, J. UGALDE, C. ALARCÓN, R. GARCÍA \& D. RODRÍGUEZ, 2014. Manual del sistema de certificación ambiental municipal. Departamento de Gestión Ambiental Local, División de Educación Ambiental, Ministerio del Medio Ambiente, Chile. Disponible en: http://www.mma.gob.cl/ educacionambiental/1319/articles-53004_ recurso_1.pdf

KANDZIORA, M., B. BURKHARD \& F. MÜLLER, 2013. Interactions of ecosystem properties, ecosystem integrity and ecosystem service indicators-A theoretical matrix exercise. Ecological Indicators, 28: 54-78.

KOSCHKE, L., C. FUERST, S. FRANK \& F. MAKESCHIN, 2012. A multi-criteria approach for an integrated land-cover-based assessment of ecosystem services provision to support landscape planning. Ecological Indicators, 21, 54-66.

LARA, A., C. LITTLE, R. URRUTIA, J. MCPHEE, C. ÁLVAREZ-GARRETÓN, C. OYARZÚN, D. SOTO, P. DONOSO, L. NAHUELHUAL, M. PINO \& I. ARISMENDI, 2009. Assessment of ecosystem services as an opportunity for the conservation and management of native forests in Chile. Forest Ecology and Management 258: 415-424.
LATERRA, P., E.G. JOBBÁGY \& J. PARUELO, 2011. Valoración de servicios ecosistémicos: Conceptos, herramientas y aplicaciones para el ordenamiento territorial. Buenos Aires: INTA.

LITTLE, C. \& LARA, A., 2010. Restauración ecológica para aumentar la provisión de agua como un servicio ecosistémico en cuencas forestales del centro-sur de Chile. Bosque, 31(3): 175178.

MAES, J., M.L. PARACCHINI \& G. ZULIAN, 2011. A European assessment of the provision of ecosystem services. JRC Scientific and Technical Reports. Luxembourg: Publications Office of the European Union.

MARTÍNEZ-HARMS, M.J. \& P. BALVANERA, 2012. Methods for mapping ecosystem service supply: a review. International Journal of Biodiversity Science, Ecosystem Services \& Management, 8(1-2):17-25.

MEA (Millennium Ecosystem Assessment), 2005. Ecosystems and Human Well-being: A Framework for Assessment. Island Press, Washington, DC. Disponible en: http://www. unep.org/maweb/en/Framework.aspx

MORALES, P., S. SCOTT, F. FERNÁNDEZ, P. GONZÁLEZ, E. VIVANCO, M. SOTO \& J. ARIAS, 2011. Valoración económica de 4 humedales altoandinos de la I región (Huasco, Coposa, Caya y Lirima). Facultad de Ciencias Agronómicas, Departamento de Economía Agraria - Servicio Agrícola y Ganadero.

NAHUELHUAL, L., P. DONOSO, A. LARA, D. NÚÑEZ, C. OYARZÚN \& E. NEIRA, 2007. Valuing ecosystem services of Chilean temperate rainforests. Environment, Development and Sustainability, 9: 481-499. 
NIEMELÄ， J., S.R. SAARELA， T. SÖDERMAN, L. KOPPEROINEN, V. YLI-PELKONEN, S. VÄRE \& D.J. KOTZE, 2010. Using the ecosystem services approach for better planning and conservation of urban green spaces: A Finland case study. Biodivers. Conserv., 19(11): 3225-3243.

OYARZÚN, C., L. NAHUELHUAL \& D. NÚÑEZ, 2005. Los servicios ecosistémicos del bosque templado lluvioso: producción de agua y su valoración económica. Revista Ambiente y Desarrollo, 20(3)-21(1): 88-95.

PLISCOFF, P. \& F. LUBERT, 2006. Diversidad de ecosistemas: Ecosistemas terrestres. En: SABALL, A. Biodiversidad de Chile: patrimonio y desafíos. Comisión Nacional del Medio Ambiente, 96-105.

RIDES, 2005. Evaluación Ecosistémica del Milenio: Bienestar Humano y Manejo Sustentable en San Pedro de Atacama, Chile. Resumen ejecutivo. Disponible en: http://www.millenniumassessment. org/documents_sga/Chile\%20Eng.\%20 Executive\%20Summary.pdf

RUCKELSHAUS, M., E. MCKENZIE, H. TALLIS, A. GUERRY, G. DAILY, P. KAREIVA, S. POLASKY, T. RICKETTS, N. BHAGABATI, S.A. WOOD \& J. BERNHARDT, 2015. Notes from the field: Lessons learned from using ecosystem service approaches to inform real-world decisions. Ecological Economics, 115: 11-21.

SUKHDEV P., H. WITTMER \& D. MILLER, 2014. The Economics of Ecosystems and biodiversity (TEEB): Challenges and Responses, in D. Helm and C. Hepburn (eds), Nature in the Balance: The Economics of Biodiversity. Oxford: Oxford University Press. Disponible en: http://img. teebweb.org/wp-content/uploads/2014/09/ TEEB-Challenges-and-Responses.pdf
TANCOIGNE, E., M. BARBIER, J.P. COINTET \& G. RICHARD, 2014. The place of agricultural sciences in the literature on ecosystem services. Ecosystem Services, 10: 35-48.

TEEB, 2010. The Economics of Ecosystems and Biodiversity for Local and Regional Policy Makers. Disponible en: http://www. teebweb.org/media/2010/09/TEEB_D2_ Local_Policy-Makers_Report-Eng.pdf

THOMSON REUTERS, 2013. Citation data. Disponible en: http:// scientific.thomsonreuters.com/imgblast/ JCRFullCovlist-2013.pdf

VÁSQUEZ, F., J.C. CASTILLA, S. GELCICH, M.A. QUIROGA, P. CARRASCO, X. PAZ \& J. RIQUELME, 2010. Evaluación económica de los activos ambientales presentes en la red de reservas marinas decretadas en el país bajo la Ley General de Pesca y Acuicultura, Informe Final. Proyecto FIP N²008-56.Universidad de Concepción. Disponible en: http://cpps. dyndns.info/cpps-docs-web/planaccion/ biblioteca/pordinario/104.Valoracion economica_reservas_marinas_Chile.pdf

WAINGER, L.A., D.M. KING, R.N. MACK, E.W. PRICE \& T. MASLIN, 2010. Can the concept of ecosystem services be practically applied to improve natural resource management decisions? Ecological Economics, 69: 978-987.

WU, J., 2013. Landscape sustainability science: ecosystem services and human well-being in changing landscapes. Landscape Ecology, 28(6), 999-1023.

YOUNG, R.F. \& S.A. WOLF, 2006. Goal attainment in urban ecology research: A bibliometric review 1975-2004. Urban Ecosystems, 9: 179-193. 